\title{
Laboreal
}

Volume $5 \mathrm{~N}^{\circ} 2$ | 2009

Varia

\section{Orientações para a avaliação das intervenções visando a prevenção dos Problemas Músculo- Esqueléticos ligados ao trabalho}

Orientaciones para la evaluación de las intervenciones sobre prevención de lesiones músculo esqueléticas (LMS)

Orientations pour l'évaluation des interventions visant la prévention des troubles musculo-squelettiques liés au travail

Orientations for the evaluation of interventions for work-related musculoskeletal disorder prevention

Fabien Coutarel, Nicole Vézina, Diane Berthelette, Agnès Aublet-Cuvelier, Alexis Descatha, Karine Chassaing, Yves Roquelaure e Catherine $\mathrm{Ha}$ Tradutor: Adelaide Nascimento e Rita Gil Mata

\section{OpenEdition}

Edição electrónica

URL: http://journals.openedition.org/laboreal/9992

DOI: $10.4000 /$ laboreal.9992

ISSN: 1646-5237

Editora

Universidade do Porto

Refêrencia eletrónica

Fabien Coutarel, Nicole Vézina, Diane Berthelette, Agnès Aublet-Cuvelier, Alexis Descatha, Karine Chassaing, Yves Roquelaure e Catherine Ha, « Orientações para a avaliação das intervenções visando a prevenção dos Problemas Músculo-Esqueléticos ligados ao trabalho », Laboreal [Online], Volume 5 No2 | 2009, posto online no dia 01 dezembro 2009, consultado o 10 outubro 2019. URL : http:// journals.openedition.org/laboreal/9992; DOI : 10.4000/laboreal.9992

Este documento foi criado de forma automática no dia 10 outubro 2019.

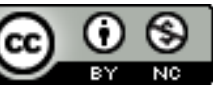

Laboreal está licenciado com uma Licença Creative Commons - Atribuição-NãoComercial 4.0 Internacional. 


\section{Orientações para a avaliação das intervenções visando a prevenção dos Problemas Músculo- Esqueléticos ligados ao trabalho}

Orientaciones para la evaluación de las intervenciones sobre prevención de lesiones músculo esqueléticas (LMS)

Orientations pour l'évaluation des interventions visant la prévention des troubles musculo-squelettiques liés au travail

Orientations for the evaluation of interventions for work-related musculoskeletal disorder prevention

Fabien Coutarel, Nicole Vézina, Diane Berthelette, Agnès Aublet-Cuvelier, Alexis Descatha, Karine Chassaing, Yves Roquelaure e Catherine $\mathrm{Ha}$

Tradução : Adelaide Nascimento e Rita Gil Mata

\section{REFERÊNCIA}

Artigo original : Coutarel, F., Vézina,N., Berthelette, D., Aublet-Cuvelier, A., Descatha,A., Chassaing, K., Roquelaure, Y. \& Ha, C. (2009). Orientations pour l'évaluation des interventions visant la prévention des troubles musculo-squelettiques liés au travail. PISTES, 11,2.

Os autores agradecem os seguintes colegas pelos seus comentários : A. Leclerc (INSERM U687) e de maneira geral, todos os actores implicados nos projectos da Angência Nacional de Pesquisa (ANR) da França “ EPID - ERGO - Action » e “TMS - SUPOR », coordenados por Annette Leclerc e Yves Roquelaure, cujos debates contribuiram para nutrir este texto.

1 A melhoria da performance das intervenções conduzidas em local de trabalho constitui um desafio maior para a prevenção dos problemas músculo-esqueléticos (PME). A 
prevenção não pode ser reduzida a uma aplicação dos modelos etiológicos disponíveis. No quadro da intervenção ergonómica, a prevenção supõe uma mobilização de actores singulares segundo formas adaptadas não definíveis antecipadamente, em contextos específicos (culturais, sociais, económicos, ...), aos quais deve igualmente adaptar-se. A melhoria das nossas acções necessita de uma melhor compreensão da passagem dos conhecimentos etiológicos gerais a acções em contextos singulares. A avaliação das intervenções visa precisamente construir uma melhor compreensão das relações entre o desenvolvimento, o contexto e os efeitos destas intervenções (Berthelette, Bilodeau \& Leduc, 2008 ; IRSST, 2008 ; Landry, Mary-Cheray \& Tayar, 2006). Este artigo pretende trazer uma contribuição à precisão de diferentes desafios e condições de avaliação das intervenções em local de trabalho.

2 Vários artigos científicos tratando da questão da avaliação das intervenções inscrevemse numa "perspectiva descendente" (por exemplo, Westgaard \& Winkel, 1997 ; Tuncel et al. 2008) : as "boas práticas" de avaliação (frequentemente originárias do ponto de vista epidemiológico) são definidas e os interventores (investigadores e/ou actuando na profissão) são convidados a segui-las tanto quanto possível. Pensamos que esta abordagem, não obstante todos os conhecimentos úteis que fornece, ignora a maior parte do tempo as especificidades da intervenção no contexto da empresa. Este artigo sublinha o interesse de uma abordagem complementar e diferente : adoptaremos uma "perspectiva inversa, de preferência ascendente". Ao colocar em evidência as características e dificuldades da intervenção em contexto real e os diferentes desafios dos interventores envolvidos nas acções de transformação, tentaremos propor orientações para favorecer práticas de avaliação eficientes, ou seja, adaptadas aos recursos dos quais dispõem os interventores a maior parte do tempo. O nosso desejo não é aqui de opor as duas abordagens de avaliação de intervenções : o leitor constatará que propomos várias vezes orientações que se nutrem de conhecimentos e de recomendações elaboradas no âmbito da primeira abordagem (descendente). Nós desejamos sublinhar neste texto a necessidade de desenvolver uma abordagem da investigação sobre a prática e a avaliação de intervenções que assuma os constrangimentos quotidianos dos interventores em saúde-trabalho e assim a ruptura epistemológica entre os modelos etiológicos e os modelos de intervenção (Daniellou, 1996). O desafio é grande porque a melhoria das condições de trabalho em todo um território não pode ser alcançada sem uma melhor compreensão do trabalho das pessoas que nele intervêm.

3 Parece necessário considerar a avaliação como uma dimensão de pleno direito da intervenção e portanto estar de acordo sobre as condições necessárias para tal. As contribuições da avaliação de uma intervenção são numerosas: guiar as decisões futuras, tirar lições das intervenções anteriores, ganhar margens de manobra para a acção seguinte; mas é preciso estar de acordo sobre os objectivos e os meios de avaliação, em coerência com os recursos negociados. Construir as modalidades de uma avaliação séria, que evite as armadilhas clássicas que evocaremos neste texto, é de certa forma uma oportunidade, enquanto interventor, de ser também avaliado, tendo em conta os diferentes desafios da intervenção.

4 Avaliar, fazer um julgamento de valor sobre uma acção visando responder a um problema, é inevitável, sobretudo da parte dos decisores, financiadores da aç̧ão. Quando os critérios de avaliação da intervenção em saúde no trabalho não foram claramente definidos, estas pessoas podem utilizar uma abordagem muito redutora da 
avaliação. Esta situação pode então conduzir a duas coisas : por um lado, a julgamentos de valor negativos ainda que o interventor possa estar satisfeito com a sua acção. Por outro lado, a avaliações positivas ainda que o interventor não esteja, ele próprio, satisfeito com o seu trabalho. Mostraremos aqui :

1. Quais são os viés clássicos relativos à avaliação de intervenções, e

2. Como os interventores no campo da saúde no trabalho podem contribuir para a construção de condições de verificação da sua eficácia.

Os desafios ligados à realização de uma avaliação são então numerosos e importantes. Veremos que eles são potencialmente de naturezas diversas: profissionais, políticos, sociais, económicos, científicos... Se o raciocínio parece clássico, as modalidades de colocação em prática são-no menos. $O$ objectivo deste texto é contribuir com elementos de reflexão para definir um quadro geral, precisando os diferentes componentes da avaliação rigorosa de um projecto em empresa : a avaliação dos efeitos da intervenção, a avaliação do seu processo e a avaliação da consideração dos efeitos potenciais do contexto no qual a intervenção foi implantada (Contandriopoulos, Champagne, Denis \& Avarguez, 2000). Tentaremos então descrever de maneira precisa as orientações relativas tanto aos objectos da avaliação quanto às modalidades desta avaliação.

\section{A avaliação dos efeitos da intervenção}

6 O que devemos avaliar em termos de efeitos de uma intervenção que visa a prevenção dos PME ? Dito de outro modo, como apreciar a ligação entre a démarche posta em prática e os efeitos observáveis?

\subsection{Os perigos de uma avaliação da intervenção através da simples avaliação da saúde dos trabalhadores}

7 A resposta não é tão simples como poderíamos supor. Na realidade, a saúde é um objecto complexo, multi-determinado. Avaliar uma intervenção em contexto de trabalho com base na evolução dos sintomas dos trabalhadores apresenta numerosas dificuldades :

1. Os efeitos da exposição sobre a saúde dos trabalhadores são diferidos no tempo (Volkoff, 2005). Isto conduz a dois questionamentos. Por um lado, quando medir os efeitos das transformações das situações de trabalho sobre a saúde a saúde dos trabalhadores? Por outro lado, avaliar simultaneamente a exposição e os seus efeitos sobre a saúde implica a hipótese implícita que a exposição actual medida é comparável à que existia na origem do estado de saúde actual... Esta hipótese é arriscada pela variabilidade dos tempos de latência segundo a natureza dos PME e o terreno sobre o qual eles se apareceram (Aublet-Cuvelier, Aptel \& Weber, 2006).

2. A validade e a fiabilidade das verbalizações dos sintomas, em particular, supõem condições de recolha rigorosas e garantias quanto ao respeito da confidencialidade dos dados recolhidos (salvaguarda, condições de acesso aos dados e restituição dos resultados, etc.). 0 contexto social pode constituir um grande viés de declaração : a expressão de uma queixa pelos salariados não é nunca inofensiva. Um plano social anunciado ou suspeito, uma situação precária com relação ao seu emprego, uma incerteza quanto à utilização das informações, prejudicam a validade e a fiabilidade das respostas dadas pelos salariados. Para contornar estas dificuldades, estão disponíveis outros meios, como a utilização do protocolo de consenso clínico SALTSA, que, efectuado por um médico, permite a recolha de 
informações a partir de uma conduta clínica sistemática repousando não só sobre a pesquisa de sintomas específicos a partir do interrogatório ao salariado, mas também sobre a realização de testes clínicos estandardizados (Sluiter, Rest \& Frings-Dresen, 2001). A entrevista semi-directiva é também um instrumento utilizável para a recolha de dados respeitantes aos sintomas, principalmente nos contextos onde os médicos do trabalho não estão presentes ;

3. Os trabalhadores já doentes antes da intervenção são menos sensíveis aos efeitos positivos da mudança: os trabalhadores nos quais os sintomas se tornaram crónicos, podem não constatar a melhoria do seu estado de saúde, mesmo que considerem as mudanças positivas. Em certos casos, eles podem apesar de tudo constatar uma estabilização, ainda que os sintomas possam ter tendência a agravar-se; em outros casos, os sintomas podem permanecer, mas pode talvez ser possível medir uma melhoria do seu estatuto funcional (por exemplo uma melhor capacidade para realizar actividades quotidianas como lavar os cabelos ou aspirar a casa) (Stock et al., 2003). o benefício da intervenção permanece, não obstante, de difícil mensurabilidade, considerando a ausência de controlo de numerosos factores que podem influenciar a patologia.

4. Os trabalhadores nos quais a frequência de sintomas é frágil, mesmo se esta frequência pudesse ser reduzida pela intervenção, não perceberão a evolução. Mesmo que eles considerem que as mudanças são positivas, eles não perceberão, portanto, nenhuma evolução do ponto de vista dos seus próprios sintomas ;

5. A intervenção é acompanhada geralmente por um aumento da sensibilização dos salariados no que diz respeito aos PME. Melhor informados sobre os sintomas, também o estão para os identificar e os exprimir. Assim, não é raro que um dos efeitos da intervenção se traduza por um aumento inicial das queixas de PME. Estas queixas não são reflexo de uma degradação das condições de trabalho ou mesmo de um aumento dos sintomas de PME, mas sim de uma melhor informação e de uma maior expressão dos salariados sobre os PME.

6. Existem interacções entre o mundo do trabalho e o mundo fora do trabalho: a saúde é sensível a outros factores que não a intervenção, seja estes ligados ao mundo do trabalho ou fora dele, num sentido positivo ou negativo. Uma mudança na esfera privada pode danificar os frágeis equilíbrios construídos pelos salariados e fazer com que estes últimos não suportem mais as suas condições de trabalho. Isto não significa que estas já não eram difíceis antes, mas que as formas de compensação que estavam inicialmente presentes não estão mais. O equilíbrio familiar (Pézé-Grenier, 2002), ou a actividade física fora do trabalho podem equivaler a formas de compensação. Todas as mudanças, como a perturbação do equilíbrio familiar, que apareça entre o início da intervenção e a medição dos seus efeitos, é um viés de história (Shadish, Cook \& Campbell, 2002).

7. A avaliação do estado de saúde só pode ser realizado junto dos trabalhadores realmente presentes no local de trabalho no momento da intervenção, mas os trabalhadores presentes na situação observada pelo interventor são os que suportaram até hoje as condições de trabalho. $O$ interventor não tem acesso directo aos que saíram da situação ou da empresa, aos que estão de baixa médica, aos que recusaram o trabalho, etc. Os trabalhadores mais afectados pelas suas condições de trabalho deixam o seu emprego. 0 efeito "trabalhador são" (Healthy Worker Effect) leva assim a uma selecção sobre a saúde da população que trabalha na empresa (Goldberg, 1995). É então possível constatar um nível muito alto de exposição e, simultaneamente, a ausência de efeito sobre a saúde dos trabalhadores presentes na empresa.

8. Qualquer que seja a qualidade das transformações realizadas, uma mudança exige uma adaptação que pode ser vivida como um momento difícil para os salariados (questionar os gestos profissionais, os estatutos sociais no seio dos colectivos, etc.) e pode acompanhar-se de novos sintomas no lançamento de novas instalações (ligadas, por exemplo, a uma situação de aprendizagem que solicita novos músculos ou coordenações musculares). No âmbito da 
avaliação, é importante seguir estes sintomas porque em muitos casos, eles serão temporários. Uma reavaliação à distância mostra-se útil para seguir a sua evolução e darlhes sentido.

9. Entre os factores profissionais implicados na etiologia dos problemas músculo-esqueléticos, alguns não poderão ser transformados devido às margens de manobra limitadas do interventor. Certos factores de risco podem assim estar fora do campo das transformações possíveis e negociáveis : o interventor pode fazer um trabalho sério no âmbito das restrições fixadas pelo empregador sem que tal se traduza por uma melhoria da saúde dos trabalhadores.

10. Quando a empresa realiza ao mesmo tempo uma intervenção e outras mudanças, os efeitos medidos dizem também respeito essas últimas. A vida da empresa não pára durante a intervenção. Outros projectos, mais ou menos coerentes com a acção de prevenção, estão em curso. É frequentemente difícil separar as coisas e um viés de história pode alterar os resultados da avaliação (Vézina et al., 2003).

8 Por estas diferentes razões, é por vezes muito difícil estabelecer uma relação directa entre a melhoria das condições de trabalho e a saúde dos salariados. Algumas dificuldades podem ser ultrapassadas por meio de dispositivos adaptados necessitando de competências importantes e específicas.

9 Ou seja, toda a melhoria de condições de trabalho não se acompanha necessariamente de um efeito benéfico mensurável sobre a saúde dos respectivos salariados. Todas as situações são possíveis numa intervenção em local de trabalho, inclusive as situações extremas onde :

- a intervenção melhora "objectivamente" a situação - ou seja, sobre os critérios mensuráveis de redução da exposição ao risco - sem melhoria da saúde dos trabalhadores,

- constatamos uma melhoria da saúde dos trabalhadores ainda que as mudanças operadas degradem objectivamente a situação de trabalho. As razões podem ser diversas : mudanças na vida privada, mudança da percepção da sua situação pelos trabalhadores - os efeitos "Hawthorn", "Pigmalião", "Placebo", abundantemente descritos na literatura podem fazer parte desta categoria.

10 Na medida em que avaliar uma acção consiste, em parte, em apreciar a sua eficácia, ou seja, a concordância entre os resultados obtidos e os objectivos obtidos, a construção dos objectivos iniciais tem uma importância muitas vezes subestimada: a que nos comprometemos enquanto interventores?

11 Em França tanto como no Québec, o indicador utilizado por numerosas empresas é o número de PME declarados e reconhecidos pela Segurança Social aquando da realização de um procedimento oficial de declaração. A conduta para obter uma indemnização, realizada por iniciativa dos salariados, é potencialmente cheia de factores em jogo e numerosos são os trabalhadores que se recusam a engrenar-se (vantagens financeiras frágeis, estatutos precários, medo de serem estigmatizados, contexto geral de desemprego, lugar no colectivo de trabalho, peso e incerteza ligados à démarche em si mesma...). O seguimento deste indicador que não resulta de uma declaração sistemática não pode por si só permitir uma avaliação da intervenção. De maneira geral, avaliar a eficácia de uma intervenção considerando como único critério a melhoria da saúde dos trabalhadores é discutível. o risco é grande para o interventor se ele for avaliado unicamente por esta base, já que numerosos factores ligados à evolução do estado de saúde ou à própria natureza dos indicadores de saúde retidos vão interagir com os 
efeitos da intervenção. Outras orientações alternativas e complementares entre si podem ser construídas :

- realizar uma medição mais fina e mais sensível da saúde dos salariados em torno dos sintomas (seguir a gravidade, a duração ou a ocorrência), e/ou da capacidade funcional dos trabalhadores (Vézina et al., 2003),

- e, de modo indissociável, interrogar a eficácia à luz dos meios ou recursos utilizados (processo de intervenção, perímetro negociado das transformações, ...). A relação entre os resultados obtidos e os recursos utilizados é chamada de eficiência (Bescos et al., 1997).

\subsection{As condições de uma avaliação do estado de saúde dos trabalhadores} abaixo especificadas) é uma das dimensões importantes e úteis na avaliação das acções de prevenção no trabalho, principalmente se essa avaliação instala na empresa um acompanhamento sistemático e regular. O questionário é o instrumento mais comum para realizar este acompanhamento. Ele permite obter, por parte dos próprios trabalhadores, dados respectivos à sua percepção do seu estado de saúde e das suas exposições profissionais.

Na maioria das vezes o questionário é auto-administrado, o que permite interrogar um grande número de salariados : o trabalhador responde sozinho às questões e envia o questionário, uma vez preenchido, a um actor encarregado de recepcionar o conjunto das respostas. 0 tratamento de dados permitirá a avaliação dos estados de saúde percebidos (assim como as exposições profissionais percebidas, já que os questionários associam frequentemente essas duas dimensões).

É importante notar os limites deste instrumento e as condições que podem ter impacto sobre os resultados obtidos. As condições temporais e operacionais da sua administração são importantes (Andersson, Karlehagen \& Jonsson, 1987; Lasfargues, Roquelaure, Fouquet \& Leclerc, 2003) :

- existem muitas variações inter e intraindividuais na descrição dos sintomas por parte dos salariados ;

- quanto mais a administração do questionário é limitada em frequência, mais a interpretação é difícil, mesmo se, por um lado, seja verdade que a análise de dados repetidos comporta outras dificuldades (por exemplo em termos de tratamento estatístico) e que, por outro lado, a escolha da frequência de administração esteja também ligada à temporalidade dos efeitos que procuramos medir ;

- as entrevistas são muito úteis para entender como os trabalhadores compreendem e utilizam o questionário numa etapa anterior à sua passagem, mas também numa etapa subsequente para validar a interpretação realizada pelos actores da avaliação.

Enfim, para optimizar a validade interna da inferência da avaliação [ $\left.{ }^{1}\right]$ as medidas antes da mudança devem, tanto quanto seja possível, visar outros grupos de trabalhadores a quem potencialmente diga respeito a mudança, a fim de :

- acompanhar a evolução da saúde dos salariados que surge em novas situações junto de um outro atelier ou serviço da empresa, tendo conhecimento do seu estado de saúde antes do projecto. A limitação do custo associado ao aumento das populações acompanhadas pode fazer-se definindo estratos nas populações [2] ; 
- acompanhar a evolução da saúde dos salariados nos quais as condições de trabalho objectivas não mudam e são inicialmente comparáveis às que são objecto de mudança. Tratase de criar um grupo controlo e seguir a evolução da percepção dos trabalhadores relativamente à sua saúde e às suas exposições. $O$ interesse e os limites do grupo controlo para a avaliação de uma intervenção já foram discutidos (Coutarel, Daniellou \& Dugué, 2005). Em certas condições, é interessante, particularmente para apreciar os efeitos da mudança nas populações vizinhas do atelier transformado e, a priori, mas apenas a priori, não relativas à mudança. Foi demonstrado que quando o grupo controlo conhece a existência do projecto [3], a situação das pessoas desse grupo pode se degradar devido à mudança da percepção deles das situações de trabalho e das novas perspectivas de evoluções que se constroem por ocasião do projecto no atelier vizinho : a saúde seria socialmente normativa, de modo que a melhoria das condições de trabalho no atelier vizinho degradaria quase automaticamente a situação dos trabalhadores não directamente relacionados com essa melhoria. Trata-se de um viés de contaminação, mais especificamente de "desmoralização rancorosa" (Shadish et al., 2002). O grupo controlo não é uma população que não beneficia de melhorias, é uma população que é privada de melhorias.

"Deve inverter-se aqui a opinião geral e convir que não é a duração de uma situação ou os sofrimentos que ela impõe que são motivos para que concebamos um outro estado das coisas onde seria melhor para todo o mundo ; pelo contrário, é a partir do dia em que pudemos conceber um outro estado das coisas que uma nova luz cai sobre as nossas queixas e sobre os nossos sofrimentos e que decidimos que eles são insuportáveis" (Sartre, 1943, p. 479, tradução livre).

O efeito inverso existe igualmente : aprendendo que não estão expostos à intervenção, os membros do grupo controlo mobilizam-se e dotam-se de outros meios para atingir os objectivos. Trata-se do viés de "rivalidade compensatória". Os vieses de contaminação reduzem a capacidade de extrapolar os resultados da avaliação a outras populações de salariados (Shadish et al., 2002).

17 As modalidades de acompanhamento das evoluções das percepções dos trabalhadores podem ser objecto de um emparelhamento individual e permitirão, por exemplo, compreender como é realizada a escolha dos salariados que ocupam novas situações (voluntários, fragilizados, sem patologia, experimentados, ...). Será interessante tentar encontrar os salariados "perdidos de vista" ao longo do processo de mudança para compreender as razões da sua transferência de atelier, de situação de trabalho, de empresa, ...

Para fazê-lo, lembremo-nos de que é indispensável seguir as recomendações e procedimentos associados aos direitos dos trabalhadores com relação à protecção dos dados relativos à sua saúde [4].

o questionário pode então constituir, sob certas condições, um instrumento de avaliação da saúde dos trabalhadores. Por exemplo, o questionário de tipo Nórdico tem uma validade suficiente para ser utilizado na vigilância dos PME (Descatha et al., 2007). Notemos todavia o interesse de afinar a interpretação dos resultados de um questionário procedendo a entrevistas junto de uma amostra de trabalhadores. $\mathrm{Na}$ realidade, em certos casos, as entrevistas podem colocar em evidência uma evolução dos sintomas que seria difícil de reparar utilizando apenas questões estandardizadas (Vézina et al., 2003 ; Vézina, Ouellet \& Major, 2009). 
20 Outras abordagens complementares de avaliação da saúde são possíveis : exame clínico estandardizado (consenso europeu SALTSA), recolha de dados médico-administrativos (baixas médicas, dores, passagens pela enfermaria...). Estes dados, de natureza e precisão diferentes, são interessantes, principalmente uma vez que desejamos obter informações relativas a situações passadas.

21 Outras utilizações do questionário são possíveis : o questionário de sintomas é frequentemente utilizado pelos interventores em entrevista semi-estruturada e pode corresponder a uma parte de pleno direito da intervenção. $O$ contacto directo com a pessoa permite ao interventor assegurar-se da compreensão exacta das questões e de tomar conhecimento de factores que podem influenciar as respostas relativamente aos sintomas. Estas informações são, evidentemente, muito preciosas para o interventor a fim de orientar as suas acções. A repetição da entrevista após as mudanças pode também permitir-lhe melhor compreender o tipo de mudança que se opera nos sintomas relatados pelos trabalhadores. Ainda que possamos admitir os limites deste tipo de dados, já que o interventor recolhe ele próprio os dados que servirão de avaliação à sua intervenção, não podemos negligenciar a importância da informação que traz este tipo de dados em termos de compreensão do vivido das pessoas entrevistadas.

\subsection{A avaliação da evolução das exposições profissionais}

22 A utilização de questionários, característica da epidemiologia, permitiu construir numerosos conhecimentos no campo dos PME e particularmente fazer reconhecer a origem profissional destes danos, por um lado, e identificar os factores de risco, por outro.

23 Às precauções citadas anteriormente quanto ao seu uso, a passagem de um questionário explorando a percepção dos trabalhadores das suas condições de trabalho pode, vantajosamente, ser acompanhado de um teste-reteste : ou seja, a passagem do mesmo questionário por uma pequena amostra de trabalhadores abrangidos, uma quinzena de dias depois da passagem inicial. Isto permitirá verificar a fiabilidade das respostas no que diz respeito às condições de trabalho [5] e à condição de acompanhar igualmente os acontecimentos que surgiram durante esses 15 dias, tendo sempre consciência dos limites associados (Stock, Fernandes, Delisle \& Vézina, 2005).

Existem numerosos questionários que são "mais ou menos validados", por exemplo no que concerne as questões relativas aos constrangimentos físicos (Stock et al., 2005). A qualidade do questionário é então determinante, e várias disciplinas, das quais a epidemiologia e a sociologia, propõem instrumentos para assegurar a validade de um questionário.

Para além disso, a qualidade dos dados recolhidos será em parte determinada pela adaptação do instrumento de base ao contexto do projecto : sem alterar ou transformar as características do instrumento, convém contudo verificar frequentemente o sentido atribuído pelo respondente às questões que lhe são colocadas no âmbito do questionário, sobretudo se ele é auto-administrado. Isto supõe uma etapa anterior à passagem, de verificação do sentido com os actores da empresa, e se necessário a construção de formulações mais adaptadas à especificidade das situações de trabalho, ou até (quando isto é possível do ponto de vista da validade de construto do questionário escolhido) a supressão de algumas questões [ $\left.{ }^{6}\right]$. 
26 Em todos os casos possíveis, as entrevistas permanecem muito úteis para entender como os trabalhadores compreendem e utilizam o questionário numa etapa anterior à passagem, mas também numa etapa subsequente para validar a interpretação realizada pelos actores da avaliação.

27 A avaliação de efeitos de uma intervenção deve então passar também por uma avaliação da evolução das exposições profissionais. É contudo incontornável situar esta evolução no ponto de vista das possibilidades de transformação negociadas pelo interventor (ver mais à frente : a avaliação do contexto da intervenção).

A natureza das diferentes exposições pertinentes do ponto de vista dos PME é-nos dada pela literatura científica internacional e particularmente da epidemiologia :

- factores biomecânicos (frequência, intensidade, duração, postura, vibrações, frio) ;

- factores psicossociais (latitude decisional, stress, apoio da hierarquia e dos colegas, exigências psicológicas da tarefa, interesse associado ao trabalho, monotonia, clima social, reconhecimento, ...) ;

- e factores organizacionais (repartição do trabalho, alternância das tarefas, implicação dos trabalhadores na concepção das suas situações de trabalho, horários e previsibilidade do tempo de trabalho, organização da produção, manutenção das instalações, conservação do material, avaliação do trabalho, etc.) [7].

Tratar-se-á menos de definir os valores absolutos por factor que os valores relativos, descrevendo a sua evolução. Com efeito, não existe norma para cada um destes factores que permita distinguir com certeza uma situação de risco de uma que não o seria : é a combinação de factores de natureza heterogénea que constrói situações de risco. A redução de constrangimentos, por factor e tanto quanto pareça possível no âmbito do projecto, permitirá qualificar essa evolução. Mas a avaliação final do projecto será objecto de um debate entre os actores implicados já que as evoluções constatadas para cada um dos factores deverão finalmente ser interpretadas em conjunto. O risco é o de cair no "todo matematizável": certas "fórmulas" propõem adicionar, subtrair ou multiplicar factores de risco entre si. Em todos os casos, estas fórmulas permanecem discutíveis e o seu resultado dificilmente interpretável: o que representa o número final quando matematizamos um ganho em termos de repetibilidade, uma perda do ponto de vista do frio e um ganho do ponto de vista da latitude decisional ? Em que é que este número desenha orientações para a melhoria das condições de trabalho ? Em numerosos casos, pudemos constatar que a obtenção de um valor único permite reduzir a complexidade da situação a fim de provocar as tomadas de decisão destinadas a atribuir recursos em termos de melhoria das condições de trabalho. Esta "utilização estratégica de fórmulas" pode mostrar-se útil deste ponto de vista.

A avaliação da evolução das exposições profissionais heterogéneas supõe a utilização de uma combinação de instrumentos associando a abordagem subjectiva (entrevistas, questionários baseados sobre o olhar trazido pelo trabalhador sobre si mesmo ou a sua situação) e abordagem objectiva (instrumentos metrológicos, análises estatísticas, observações por um expert das situações reencontradas pelos trabalhadores, ...). A natureza dos instrumentos a associar depende dos desafios e constrangimentos específicos a cada intervenção. A diversidade de dados permitirá a consideração de informações complementares umas às outras, para interpretar resultados convergentes, diferentes, eventualmente contraditórios e assumir a complexidade das situações reais de trabalho. 


\subsection{A abordagem pela actividade e o trabalho : os questionamentos complementares}

31 Os questionamentos e medições que podem conduzir a apreciar a evolução das exposições podem situar-se a diferentes níveis : isto pode fazer-se ao nível da saúde, das exposições aos factores de risco, como acabámos de precisar. Pode fazer-se igualmente, e de modo complementar, a um nível mais geral e mais transversal em torno de questionamento centrados no trabalho.

32 "A ergonomia da actividade" (Laville, 2001; Daniellou, 2005) oferece então recursos nesse sentido em virtude da especificidade da sua abordagem. Poderemos aqui recordar o carácter fundador da distinção entre trabalho real e trabalho prescrito no duplo nível da tarefa e da actividade, atravessado das noções de variabilidades e de regulações no trabalho (Guérin, Laville, Daniellou, Duraffourg \& Kerguelen, 1997 ; Falzon, 2004).

As principais características que esta abordagem retém da actividade de trabalho são as seguintes (Daniellou \& Rabardel, 2005) :

1. a actividade de trabalho é finalizada por um ou vários objectivos que não são sempre evidentes ;

2. a relação entre sujeito e objecto é mediada pelos dispositivos técnicos, pelos esquemas individuais e a sua organização, eles mesmos marcados pelo contexto social, cultural e histórico do sujeito ;

3. a actividade é sempre singular;

4. a actividade é portadora da sua história ;

5. a actividade comporta sempre uma dimensão colectiva, o outro podendo estar presente através de instrumentos, utensílios, regras ou procedimentos ;

6. a abordagem da actividade deve ser intrínseca, isto é, capaz de compreender as racionalidades que motivam as regulações postas em prática pelos trabalhadores $\left[{ }^{[}\right]$;

7. enfim, a actividade é integradora, isto é, a sua construção responde a um grande número de determinantes.

34 Assim, uma tal abordagem do trabalho tentará articular as dimensões sistémicas, dinâmicas, históricas e culturais desta actividade, respeitando a diversidade dos indivíduos e as suas racionalidades próprias. Colocando a ênfase nos determinantes dos factores de risco, alvos privilegiados da acção de transformação do local de trabalho, a ergonomia da actividade define os PME como um sintoma, entre outros possíveis, de um disfuncionamento organizacional geral do sistema de produção de bens ou de serviços, onde o deficit de margens de manobra dos diferentes actores da organização parece central (Vézina, 2001; Coutarel, 2004). É também importante recordar que a acção sobre certos determinantes de factores de risco está fora do alcance do interventor e releva de acções colectivas de uma outra natureza (Caroly et al., 2008).

Lançar o debate nestes termos no campo da avaliação de acções de prevenção dos PME em contexto profissional, conduz-nos a propor questionamentos organizados em torno das margens de manobra (Vézina, 2001; Coutarel, 2004) de diferentes actores da organização ["], para dispor de outros indicadores de avaliação das intervenções. Por exemplo :

No que diz respeito à organização do trabalho :

- as diferentes prescrições das quais os trabalhadores são o alvo, serão negociáveis em caso de dificuldade ? Serão compatíveis entre si ? 
- quais são as possibilidade para os trabalhadores de regular o desenrolar da sua actividade de trabalho (procedimentos, modos operatórios, gestos profissionais, cadência, ...) ?

- No que diz respeito às capacidades individuais e colectivas para fazer face :

- a mudança é acompanhada pelo desenvolvimento da actividade dos trabalhadores (participação dos salariados no projecto, formação, possibilidade de entreajuda, conhecimento do sistema de produção no seu todo, ...) ?

- o projecto contribuiu para o reforço do colectivo de trabalho de modo que ele se construa como recurso para cada trabalhador?

No que diz respeito aos factores a montante e jusante das situações implicadas pelas transformações :

- as transformações realizadas têm impactos sobre outras situações de trabalho situadas a montante ou a jusante ? De que naturezas são estes impactos no seio da estrutura?

- o projecto tem um impacto também sobre os actores externos à empresa (institucionais, clientes, fornecedores) ? De que naturezas são estes impactos no exterior da estrutura?

No que diz respeito aos instrumentos de gestão da vida da empresa :

- o projecto permitiu a evolução dos instrumentos de gestão utilizados ?

- os novos instrumentos e/ou indicadores provenientes da análise do trabalho real foram implementados? De que naturezas são essas mudanças?

No que diz respeito à concepção de instrumentos e espaços de trabalho :

- como foram tidas em conta, na concepção das situações de trabalho, as variabilidades das situações de trabalho encontradas?

- os instrumentos e espaços de trabalho podem ser adaptados ou mudados facilmente? Eles são adaptáveis?

No que diz respeito aos conceptores das organizações :

- o projecto fez a demonstração, junto dos decisores e dos quadros, do interesse da participação dos trabalhadores nos projectos de concepção?

- o projecto permitiu melhorar as condições de trabalho daqueles que tê a missão de organizar o trabalho dos outros (por exemplo, os quadros)?

- o projecto permitiu aos decisores e aos quadros conhecer melhor a realidade dos constrangimentos de trabalho (mudança das representações)?

No que diz respeito à organização de projectos :

- o projecto permitiu transformar as práticas as empresa em termos de condução de projecto (estruturação social e técnica dos projectos, conduta, instrumentos, ...) ?

- os diferentes actores implicados pelo projecto são considerados segundo as modalidades diferenciadas e explicitadas (missões, modalidade, temporalidades), fundadas sobre os conhecimentos actuais em termos e condução de projecto (Daniellou, 2004) ?

No que diz respeito ao poder de decisão da direcção do estabelecimento :

- o projecto contribuiu para o aumento da autonomia da qual dispõe a direcção nas decisões relativas à gestão do estabelecimento (a condução da mudança, a concepção, as modalidades de organização do trabalho, a gestão da produção), em comparação com os seus colaboradores (accionistas, grupo ao qual o estabelecimento pertence, clientes, fornecedores)?

43 Os questionamentos ligados à avaliação dos efeitos de uma intervenção são potencialmente numerosos. 

dos projectos sobre a performance económica: será que há ganhos de produtividade associados à melhoria das condições de trabalho ? O que são custos directos, indirectos ou ocultos? Qual é o retorno de investimento esperado/alcançado? Como se caracteriza a curto prazo e a médio prazo (produtividade, qualidade, imagem social, fidelização do pessoal, ...) ? Como vimos para a questão da saúde, é frequentemente difícil estabelecer um laço directo entre a performance económica e as mudanças operadas : a performance económica depende de numerosos factores não considerados pela intervenção? Tanto a saúde como a performance constituem alvos indirectos da intervenção ergonómica. No âmbito da avaliação de intervenções, é então necessário juntar outros indicadores. Propomos que as margens de manobra dos salariados possam constituir uma categoria de indicadores pertinentes e complementares.

dicadores utilizados para a avaliação de uma intervenção são determinados pelos modelos etiológicos mobilizados, mais também pelos modelos de transformação explicitados ou não pelos interventores. Para além dos efeitos da intervenção, o processo de mudança e de transformação constitui, assim, um outro alvo importante da avaliação.

\section{A avaliação do processo e a consideração do contexto de mudança}

\subsection{A avaliação do processo de mudança}

O desenrolar da empreitada de avaliação merece ser documentado (Bellemare, Duval, Baril-Gingras, Ross \& Poulin, 2008; Baril-Gingras, Bellemare, Ross \& Poulin, 2008; Vézina \& Tougas, 2008). Do ponto de vista da avaliação, o que distingue uma intervenção na empresa e uma intervenção experimental em laboratório é o carácter irredutível da diferença entre as modalidades inicialmente previstas e as modalidades realizadas (Rossi \& Freeman, 1999). O status do modelo na intervenção ergonómica, como em qualquer outra intervenção em meio vivo, deve ser determinado. 0 modelo de intervenção que permite de organizar a acção não deve ser simplesmente aplicado : ele constitui uma base inicial e operante $\left[{ }^{10}\right]$, que deve ser ajustada às singularidades dos contextos e às suas dinâmicas nem sempre controláveis. Trata-se de ajustar o "tratamento", cuja eficácia demonstrada, pelas experiências anteriores, permitiu estabilizar os conhecimentos gerais relativos aos fenómenos e às acções, a fim de propor o "cuidado", prova de que a especificidade das situações e dos actores foram levadas em consideração. Neste sentido, aplicar de maneira estrita um modelo geral de intervenção a um contexto cuja singularidade não é considerada como um dado pertinente apresenta inúmeros riscos do ponto de vista das possibilidades de melhoria das condições de trabalho.

Esta questão conduz a uma ideia de uma diferença possível (e frequente) entre o modelo de intervenção inicial e a intervenção realizada. Esta diferença não constitui necessariamente um limite, mas :

- como a característica da acção em meio vivo é complexa

- e nós só notamos algumas especificidades no decorrer do processo,

- estas mesmas susceptíveis à variabilidades,

- devemos adaptar-nos constantemente para que a intervenção seja performante. 

em si, é indispensável acompanhar, durante toda a intervenção, o seu contexto e a sua evolução eventual. (Contandriopoulos et al., 2000 ; Berthelette et al., 2008). É preciso ser capaz de identificar se outros acontecimentos, além da intervenção, podem ajudar na explicação dos resultados obtidos. Pode tratar-se neste caso de viés ou ainda de factores que interagem com a intervenção na produção dos efeitos observados.

51 As informações a recolher são de natureza diversificada : actores implicados, relações contratuais cliente-fornecedor, situação económica, legislativa, social... A escolha das informações a serem recolhidas depende do tipo de intervenção e das características dos ambientes de trabalho nos quais a intervenção é implementada (Bellemare et al., 2008).

52 É importante insistir aqui sobre a necessidade de recolher dados actuais. Na realidade, por razões diversas, a memória individual e colectiva é pouco confiável :

- esquecimentos,

- escolhas inconscientes,

- acontecimentos considerados significativos em função do momento em que a pergunta foi feita e não necessariamente em função do momento de ocorrência,

- racionalização a posteriori,

- mecanismos de auto-justificação.

53 É importante recolher esses dados actuais através de ferramentas validadas (jornal de bordo, por exemplo) e que permitem uma recolha o mais sistemática e exaustiva possível. Na realidade, os acontecimentos podem não parecer significativos no momento em que eles ocorrem, mas a influência deles na intervenção pode ser percebida a longo termo.

54 A intervenção em equipa é mais favorável à recolha de dados relativos às estratégias dos interventores e aos acontecimentos julgados significativos, pois a colaboração organiza-se principalmente em torno de momentos explícitos e organizados como reuniões, discussões e debates, que têm a vantagem de poderem ser gravados (Petit, Querelle \& Daniellou, 2007). Quando o interventor intervém sozinho, é possível negociar para que ele preencha um jornal de bordo, que irá permitir-lhe anotar as etapas do processo de intervenção e os elementos do contexto. As condições devem ser bem preparadas com o interventor pois preencher quotidianamente um jornal de bordo pode ser muito dispendioso. É preciso que o avaliador justifique et/ou compense este custo temporal. Além disso, a natureza do jornal é certamente determinante com relação às informações que serão recolhidas. 


\section{Do interesse da avaliação do projecto visando a prevenção dos PME às condições dessa avaliação}

Este texto sublinha ao mesmo tempo o interesse da avaliação das intervenções em matéria de prevenção dos PME e a complexidade desse tipo de conduta. Várias modalidades de realização de uma avaliação merecem serem aqui lembradas.

\subsection{Avaliar uma intervenção é um projecto em si}

56 Avaliar um projecto constitui um desafio de grande porte por todos os motivos anteriormente citados. Trata-se igualmente de uma questão complexa, cujo acompanhamento por especialistas do assunto é pertinente. Isto permite apreciar em que nos envolvemos, deixar claras as condições de realização, evitar os defeitos clássicos e principalmente evitar perder tempo e energia com a implementação de uma avaliação que, por causa de condições desfavoráveis, não culminará em nenhum resultado analisável nem rigoroso. A avaliação de um projecto constitui um projecto em si : um projecto dentro do projecto. Trata-se de reunir as condições necessárias: recursos, tempo, competência, aceitabilidade, antecipação...

Vimos que avaliar um projecto supõe a recolha de dados diversos e heterogéneos assim como a implicação de uma diversidade de actores que devem debater para construir colectivamente uma análise sistémica a partir dos resultados identificados. Avaliar supõe assim construir as condições de uma discussão organizada, onde todos têm voz, em torno dos efeitos heterogéneos da mudança. Uma discussão organizada supõe ferramentas, objectos construídos para favorecer o debate.

Esta "disputa" onde todos têm voz conduz à questão : "quem avalia a mudança ?". o interventor com certeza não é o único que pode estar implicado e uma dimensão da intervenção concerne a identificação dos actores pertinentes a serem solicitados. Esses actores, indivíduos ou grupos tocados pela intervenção e sua avaliação, são potencialmente numerosos : o interventor, a direç̧ão, os quadros, os operadores, os parceiros institucionais, os clientes, os fornecedores... As modalidades de mobilização desses actores devem ser construídas com eles. A avaliação é assim um projecto que supõe uma construção social.

\subsection{Avaliar uma intervenção supõe uma organização temporal}

59 A avaliação de um projecto organiza-se no tempo, e isto segundo níveis diferentes. Primeiramente, se o modelo de avaliação antes/depois é interessante, deve-se estar atento pois :

1. a avaliação do projecto nunca é totalmente "antes". Na realidade, o início do projecto e a presença do interventor já constituem uma mudança importante na empresa. Já é resultado de um importante trabalho de discussões, de negociações, de eventuais transacções que não podem deixar indiferentes os interlocutores da empresa. A mudança das suas representações, que conduziu ao projecto das PME, já é um efeito muito importante da intervenção e das acções anteriores; esses decisores não conduzem hoje a mudança da mesma forma que o fizeram ontem. Nós conhecemos bem a importância das representações dos decisores na construção, culminando na concepção das situações de trabalho, 
potencialmente geradoras de PME. A utilização de certos indicadores (dados médicos, de exposição, de gestão dos recursos humanos, de produção...) podem, no entanto, em muitos casos, contribuir a caracterizar o "antes".

2. a avaliação "depois" não deve ser feita nem muito cedo, nem muito tarde. Com efeito, muito cedo depois da mudança, a avaliação da saúde dos trabalhadores pode mostrar um aumento das queixas. Nós já observamos que o aparecimento de novas queixas é independente da qualidade das transformações operadas e parece ser inerente a toda mudança (Coutarel, 2004) - daí a importância de avaliações objectivas das contraintes. Muito tarde, a avaliação corre o risco de medir efeitos que não estão ligados ao projecto, mas a acontecimentos ocorridos entre o fim do projecto e o momento da avaliação - daí a importância do acompanhamento do contexto. ajustada à temporalidade do projecto. Na realidade, se usarmos o exemplo relativo à implantação de uma mudança que duraria 3 meses, seguida de um aumento progressivo do funcionamento das instalações de 3 meses adicionais :

- se considerarmos a totalidade do projecto, talvez não seja oportuno fazer uma medição nos 6 meses em questão ;

- se considerarmos que, como as intervenções têm proximidade temporal, os salariados modificam por antecipação suas percepções da situação. Nesse sentido, talvez não seria oportuno realizar medições nas semanas que antecedem as transformações ;

- Se considerarmos i) que o prazo de estabilização dos novos métodos de trabalho e do funcionamento das novas situações de trabalho, ii) que o tempo de aquisição de novos gestos, iii) que o tempo de constituição dos colectivos de trabalho, etc. são mais longos do que a planificação do aumento progressivo da produção, pode-se julgar inoperante a realização de novas medições durante os 3 meses seguintes ao aumento progressivo do funcionamento da instalação. No caso contrário, é importante estar atento quanto à interpretação dos resultados. 
avaliação retidos [12]. Enfim, aconselha-se estar atento quanto à regularidade dos espaços temporais entre os diferentes momentos da avaliação de um mesmo critério, e se a avaliação mobiliza diversas ferramentas, é importante que as diferentes medições sejam feitas num período relativamente curto.

A natureza dos componentes necessários e suficientes a serem documentados na démarche da intervenção, visando assegurar o seu acompanhamento e permitindo a sua avaliação, continua a ser uma questão importante para os pesquisadores e deverá tornar-se um objecto consensual. Este texto visa sublinhar a complementaridade das avaliações da saúde e das avaliações do trabalho, tendo em vista os limites intrínsecos de cada um desses tipos de avaliação.

67 Avaliar um projecto revela inúmeros interesses por uma diversidade de actores. A avaliação do projecto é uma responsabilidade compartilhada. Isso não significa avaliar somente a acção do interventor, como muitas vezes se deseja. Significa avaliar os efeitos da mobilização de todos os actores implicados ou não na conduta. Querer especificar a responsabilidade de um interventor isoladamente na avaliação de um projecto não parece fazer sentido para nós : por um lado, as abordagens participativas supõem colaborações diversas e a recusa de alguns ao envolvimento no projecto é, de uma maneira ou de outra, uma forma de implicação no projecto; por outro lado, a transformação das situações de trabalho e a prevenção de PME continuam, no fim de contas, a ser uma responsabilidade do empregador. $O$ facto deste não se envolver na démarche deve assim ser analisado tanto do ponto de vista do modelo do interventor, como do ponto de vista do empregador (contexto). A confiança que este fornece ao interventor, deixando-o agir, deve ser um elemento da avaliação. A condução de projecto em ergonomia, principalmente no meio industrial e da arquitectura, sublinha desde há muito tempo os efeitos negativos da não implicação do empregador sobre o sucesso do projecto (Martin, 2004). Este aspecto deve igualmente constituir um elemento de avaliação da intervenção de prevenção de PME.

Os critérios potenciais da avaliação da intervenção são assim múltiplos e de natureza heterogénea. Isto significa que "a contribuição global" de uma intervenção não se mede, aprecia-se, mesmo se os critérios, considerados isoladamente, possam ser medidos. Classicamente, o critério dos sintomas e da saúde é escolhido, mas, como foi visto neste artigo, não é suficiente. Introduzir na avaliação critérios relativos às margens de manobra dos diferentes actores da estrutura permite estender o questionamento até aos determinantes dos factores de riscos identificados na literatura. Com certeza isto também contribui para imaginar de maneira mais operacional as possibilidades de melhoria das situações.

Não podemos improvisar a apreciação, de maneira pertinente, do desenrolar do projecto e dos seus efeitos. Várias condições devem estar reunidas e devem ser negociadas com muita antecedência para a apreciação (e não as mensurações) das relações entre os efeitos identificados, o processo conduzido e o contexto do projecto. Reunir essas condições, ou aproximar-se o melhor possível delas [ $\left.{ }^{13}\right]$, é aproximar-se de uma avaliação séria do projecto e dos interventores, que não podem ser dissociados dos outros actores. Avaliar o projecto constitui um projecto no projecto. A avaliação do projecto supõe, entre outras coisas, a construção de espaços e lugares dedicados ao debate colectivo, segundo lógicas heterogéneas, efeitos identificados. Criar esses espaços de debate, sob o pretexto da avaliação, contribui igualmente para a 
transformação das representações dos actores implicados. Nessa perspectiva, avaliar é também transformar o trabalho.

\section{BIBLIOGRAFIA}

Aublet-Cuvelier, A., Aptel, M. \& Weber, H. (2006). The dynamic course of musculoskeletal disorders in an assembly line factory. International Archives of Environmental Health, 79, 578-584.

Andersson, K., Karlehagen, S. \& Jonsson, B. (1987). The importance of variations in questionnaire administration. Applied Ergonomics, 18, 229-232.

Baril-Gingras, G., Bellemare, M., Ross, J. \& Poulin, P. (2008). Un outil de bilan de l'intervention : effets intermédiaires, processus et changements chez les acteurs du milieu de travail. Actes du 2ème Congrès Francophone TMS (http://www.irsst.qc.ca). IRSST : Montréal.

Bellemare, M., Duval, L., Baril-Gingras, G., Ross, J. \& Poulin, P. (2008). Des outils pour analyser les aspects socio-organisationnels des interventions. Actes du 2ème Congrès Francophone TMS (http:// www.irsst.qc.ca). IRSST : Montréal.

Berthelette, D., Bilodeau, H. \& Leduc, N. (2008).Pour améliorer la recherche évaluative en santé au travail. Santé publique, 20, 3, 171-179.

Berthelette, D., Leduc, N., Bilodeau, H., Durand, M.-J., Faye, C., Loignon, C. \& Lagacé, M.-J. (2006). Analyse d'un programme de formation visant la prévention primaire des maux de dos chez le personnel soignant des centres hospitaliers du Québec. Montréal : Institut de recherche Robert-Sauvé en santé et en sécurité du travail. (http://www.irsst.qc.ca/).

Bescos, P-L., Dobler, P., Mendoza, C., Naulleau, G., Giraud, F. \& Lerville, A. (1997). Contrôle de gestion et management. Paris : Editions Montchrestein.

Bonnet, M. (2006). Lien en GRH et contrôle de gestion dans le domaine de la santé au travail. Exemple d'une recherche intervention centrée sur la rpévention des troubles musculo-squelettiques. Actes du XVII Congrès de l'AGRH. Lille, France.

Campbell, D. T. \& Stanley, J.C. (1963). Experimental and Quasi-Experimental Designs for Research on Teaching. In N. L. Gage (ed.), Handbook of Research on Teaching. Chicago: Rand McNally.

Caroly, S., Coutarel, F., Escriva, E., Roquelaure, Y., Schweitzer, J-M. \& Daniellou F. (coord.) (2008). La prévention durable des TMS, Quels freins? Quels leviers d'action? Recherche-action 2004-2007. Rapport de recherche pour Direction Générale du Travail. http://www.anact.fr/

Clot, Y. (1999). La fonction psychologique du travail. Paris : PUF.

Coutarel, F. (2004). La prévention des troubles musculo-squelettiques en conception : quelles marges de manœuvre pour le déploiement de l'activité ? Laboratoire d'Ergonomie des Systèmes Complexes, Université Bordeaux 2, Alection Thèses \& Mémoires.

Coutarel, F., Daniellou, F. \& Dugué, B. (2005). La prévention des troubles musculo-squellettiques : des enjeux épistémologiques. Activités, 3, 2, 3-19. 
Contandriopoulos, A.P., Champagne, F., Denis, J.L. \& Avarguez, M.C. (2000). L'évaluation dans le domaine de la santé : concepts et méthodes. Revue d'Epidémiologie et de Santé Publique, 48, 517-539.

Daniellou, F. (2005). The French-speaking ergonomist's approach to work activity; crossinfluences of field intervention and conceptual models. Theoretical Issues in Ergonomics Science, 6, 5, 409-427.

Daniellou, F. (dir.) (1996). L'ergonomie en quête de ses principes. Toulouse : Octarès.

Daniellou, F. (2004). L'ergonomie dans la conduite de projets de conception de systèmes de travail. In P. Falzon (dir.) (2004), Ergonomie (pp. 359-373). Paris : PUF.

Daniellou, F. \& Rabardel, P. (2005). Activity-oriented approaches to ergonomics: some traditions and communities. Theoretical Issues in Ergonomics Science, 6, 5, 353-357.

Descatha, A., Roquelaure, Y., Chastang, J.F., Evanoff, B., Melchior, M., Mariot, C., Ha, C., Imbernon, E., Goldberg, M. \& Leclerc, A. (2007). Validity of Nordic-style questionnaires in surveillance of upper-limb work-related musculoskeletal disorders. Scandinavian Journal of Work Environment and Health, 33, 58-65.

Falzon, P. (dir.) (2004). Ergonomie. Paris : PUF.

Guérin, F., Laville, A., Daniellou, F., Duraffourg, J. \& Kerguelen, A. (1997). Comprendre le travail pour le transformer. Editions de l'ANACT.

Goldberg, M. (1995). L'épidémiologie sans peine. Paris : Frison Roche.

IRSST (2008). Actes du 2ème Congrès Francophone sur les TMS. Montréal.

Landry, A., Mary-Cheray, I. \& Tayar, E. (2006). Proposition for evaluations of musculoskeleteal disorders preventive intervention. Proceedings of IEA 16th congress, Maastricht.

Lasfargues, G., Roquelaure, Y., Fouquet, B. \& Leclerc, A. (2003). Pathologie d'hypersollicitation périarticulaire des membres supérieurs. Paris : Ed. Masson.

Laville, A. (2001). Repères pour une histoire de l'ergonomie francophone. Actes Congrès Self-Ace, 2001, Montréal.

Martin, C. (2004). L'ergonome dans les projets architecturaux. In P. Falzon (dir.), Ergonomie (pp. 421-435). Paris : PUF.

Petit, J., Querelle, L. \& Daniellou, F. (2007). Quelles données pour la recherche sur la pratique de l'ergonome? Le Travail Humain, 4, 70, 391-411.

Pezé-Grenier, M. (2002). Le deuxième corps. Paris : La Dispute.

Rossi, P.H. \& Freeman, H.E. (1999). Evaluation: A Systematic Approach. Beverly Hills, CA: Sage Publications.

Sartre, J-P. (1943). L'être et le néant. Paris: Editions Gallimard.

Scheirer, M.A. (1987). Program Theory and Implementation Theory: Implications for Evaluators. In Using Program Theory in Evaluation, Bickman L (ed), New Directions for Program Evaluation, 33, 59-76.

Shadish, W.R., Cook T.D. \& Campbell D.T. (2002). Experimental and Quasi-Experimental Designs for Generalized Causal Inference. Florida: Houghton Mifflin Company.

Sluiter, B.J., Rest, K.M. \& Frings-Dresen, M.H. (2001). Criteria document for evaluating the workrelatedness of upper-extremity musculoskeletal disorders. Scandinavian Journal of Work Environment and Health, 27, 1, 1-102. 
Stock, S.R., Loisel, P., Durand, M.J., Streiner, D., Tugwell, P., Reardon, R., Lemaire, J., Boucher, M., Darzins, S., Dilworth, P. \& Goudreault, N. (2003). IDVQ : l'Indice d'impact de la douleur au cou et aux membres supérieurs sur la vie quotidienne. Développement et validation d'une nouvelle mesure de l'état de santé pour les travailleuses et travailleurs atteints de lésions musculo-squelettiques du cou et des membres supérieurs. Études et Recherches R-355, IRSST.

Stock, S.R., Fernandes, R, Delisle, A. \& Vézina, N. (2005). Reproducibility and validity of worker's self reports of physical work demands. Scandinavian Journal of Work Environment and Health, 31, 6, 409-437.

Tuncel, S., Genaidy, A., Shell, R., Salem, S., Karwowski, W., Darwish, M., Noel, F. \& Singh, D. (2008). Research to pratice: Effectiveness of Controlled Workplace Interventions to Reduce Musculoskeletal Disordersin Manufacturing Environment - Critical Appraisal and Meta-Analysis. Human Factors and Ergonomics in Manufacturing, 18, 2, 93-124.

Vézina, N. (2001). La pratique de l'ergonomie face aux TMS : ouverture à l'interdisciplinarité. Actes du Congrès SELF-ACE, 44-60.

Vézina, N., Stock, S.R., Simard, M., Saint-Jacques, Y., Boucher, M., Lemaire, J. \& Trudel, C. (2003). Problèmes musculo-squelettiques et organisation modulaire du travail dans une usine de fabrication de bottes - Phase 2 : Étude de l'implantation des recommandations. Alection Études et Recherches - IRSST, R-345. (http://www.irsst.qc.ca/).

Vézina, N. \& Tougas, G. (2008). De l'intervention de mobilisation à l'intervention ergonomique et au suivi des recommandations : quels indicateurs pour un suivi du processus. Actes du 2ème Congrès Francophone TMS (http://www.irsst.qc.ca/). IRSST : Montréal.

Vézina, N., Ouellet, S. \& Major, M-E. (2009). Quel schéma corporel pour la prévention des troubles musculo-squelettiques ? Corps, 6, 61-68.

Volkoff, S. (2005). Les approches diachroniques des relations santé, travail. Actes du séminaire CREAPT, les évolutions de la santé au cours de la vie professionnelle : altération, préservation, construction( $\left.\mathrm{n}^{\circ} 27\right)$. Noisy-Le-Grand : CEE (Al. Rapport de Recherche). http://www.ceerecherche.fr/fr/rapports.htm

West, S.G., Duan, N., Pequegnat, W., Gaist, P., Des Jarlais, D.C., Holtgrave D., Szapocznik, J., Fishbein, M., Rapkin, B., Clatts, M., \& Dolan Mullen, P. (2008). Alternatives to the Randomized Controlled Trial. American Journal of Public Health, 8, 1359-1366.

Westgaard, R.H. \& Winkel, J. (1997). Ergonomic intervention research for improved musculoskeletal health: a critical review. International Journal of Industrial Ergonomics, 20, 463-500.

Wisner, A. (1972). Le diagnostic en Ergonomie ou le choix des modèles opérants en situation réelle de travail. Conférence de Cardiff au Congrès de l'Ergonomics Research Society in A. Wisner, (1995), Réflexions sur l'ergonomie (1962-1995) (pp. 79-102). Toulouse : Editions Octarès.

\section{NOTAS}

1. Importa precisar que o recurso a grupos controlo não elimina inteiramente os riscos de viés podendo afectar a validade interna das inferências, ou seja, o risco de concluir erradamente que uma intervenção é ou não eficaz. Desde 1963, Campbell e Stanley propuseram diferentes estratégias, sempre úteis, para reduzir este viés. West et al. (2008) propuseram recentemente dispositivos interessantes a este propósito. 
2. Por exemplo, $100 \%$ dos trabalhadores respeitantes à mudança, $50 \%$ dos trabalhadores do atelier potencialmente fornecedor principal de operadores novos, $10 \%$ em outros ateliers, com tiragem ou sorteio sistemático das populações...

3. O que é efectivamente muitas vezes o caso, já que, para seguir as populações comparáveis do ponto de vista das suas características e das suas evoluções, o grupo controlo está frequentemente na mesma empresa. Quando não está, existe a possibilidade de que a comparação das populações seja mal feita.

4. Em França, uma autorização da Comissão Nacional da Informática e das Liberdade, dada após parecer do Comité Consultivo sobre o Tratamento da Informação em Matéria de Investigação no domínio da Saúde.

5. Com efeito, o teste-reteste não deve dizer respeito às questões relativas aos sintomas, que, esses, podem ter evoluído sem que isso implique um problema de fiabilidade.

6. Por exemplo, questões como: “é funcionário?", "trabalha durante a noite?" são totalmente inapropriadas a certos contextos e têm como efeito perturbar o respondente ou mudar o seu relato na passagem auto-administrada. A falta de sentido de certas questões pode conduzir a uma lassidão que se repercutirá na qualidade das respostas às questões seguintes.

7. Um trabalho de precisão e de consenso por parte dos investigadores parece, apesar de tudo, indispensável para clarificar o que é subentendido nestes termos e para além de uma denominação que recubra aspectos frequentemente diferentes quando comparamos os trabalhos. Não entraremos aqui neste debate.

8. Os trabalhos convergentes surgidos de disciplinas vizinhas vêm enriquecer este olhar. Citemos, por exemplo, Clot (1999), que destaca que a actividade não se resume ao que é visível ou observável, sendo grandemente marcada por arbitragens realizadas entre as diferentes formas de prescrição, quer sejam conscientes ou não.

9. Notemos que certos questionamentos relativos aos factores de risco organizacionais aproximam-se dos que são propostos aqui.

10. Se Wisner (1972) falava do termo «modelos operantes»em relação a ligações construídas, através da análise ergonómica do trabalho entre o trabalhador e os determinantes da situação de trabalho, nós consideramos pertinente usar essa formulação para falar dos modelos mobilizados pelo ergónomo para transformar as situações de trabalho.

11. Ver, por exemplo, um estudo recente sobre um programa ergonómico de prevenção das dores de coluna. (Berthelette et al., 2006).

12. Notemos que a temporalidade guia as interpretações e análises possíveis. Por exemplo, se utilizarmos um questionário sobre a saúde, a sua adaptação pode ser necessária no que concerne algumas formulações, quando a temporalidade não é coerente com o projecto. Por exemplo, a pergunta incluindo "durante os últimos 12 meses" pode ser modificada se os 12 últimos meses não condizem com a temporalidade do projecto: se algumas situações só existem há 6 meses, se uma mudança ocorreu... Por outro lado, é importante conservar a mesma ferramenta de investigação antes e depois da mudança. As modificações devem assim serem realizadas logo no início da mudança.

13. Nós não queríamos contribuir com o discurso de que se todas as condições não forem reunidas, não podemos avaliar o projecto. A advertência refere-se aos eventuais limites que possam aparecer. Dito isto, é da responsabilidade dos interventores e/ou dos empregadores «determinar se o esforço vale a pena», tendo em conta as orientações aqui apresentadas. 


\section{RESUMOS}

A melhoria das práticas de intervenção constitui hoje um desafio no campo da prevenção dos Problemas Músculo-Esqueléticos (PME) ligados ao trabalho. 0 desenvolvimento de conhecimentos respeitantes à intervenção em contexto de trabalho constitui dessa forma um desafio em termos de investigação, que convoca a temática da avaliação das intervenções. A pesquisa internacional no campo dos PME concentra grandemente os seus esforços e recomendações na avaliação dos efeitos das intervenções considerando o estado dos sintomas dos trabalhadores afectados e/ou a evolução das exposições.Neste texto, mostramos logo de início que esta orientação supõe a reunião de um certo número de condições importantes, frequentemente difíceis de reunir e geralmente subestimadas. Propomos que sejam levados em conta factores relativos à actividade de trabalho e às margens de manobra dos salariados - como critérios complementares da avaliação - sublinhando sempre a necessidade de esforços para torná-los operacionais. Num segundo momento, recordamos que a avaliação dos efeitos de uma intervenção é insuficiente para o desenvolvimento de conhecimentos sobre a intervenção em si : este supõe necessariamente a descrição precisa do processo de intervenção e a consideração do contexto do seu desenvolvimento. Enfim, a discussão sublinha o facto de que, na nossa perspectiva, a avaliação das intervenções constitui um projecto em si, que contribui directamente para a transformação do trabalho.

Mejorar las prácticas de intervención constituye hoy en día un desafío en el campo de la prevención de lesiones músculo esqueléticas (LMS) relacionadas con el trabajo. El desarrollo de conocimientos sobre la intervención en las empresas es un desafío para la intervención dirigida a su evaluación. La investigación internacional en el campo de las LMS concentra sus trabajo y recomendaciones sobre la evaluación de los efectos de las intervenciones mediante la evaluación de los síntomas de salud o de las exposiciones. En este texto mostramos en primer lugar, que esta orientación supone que se reúnan un cierto número de condiciones importantes, generalmente subestimadas y a menudo difíciles de reunir. Proponemos que se tomen en cuenta, en tanto criterios complementarios de la evaluación, los indicadores relacionados a la actividad de trabajo y a los márgenes de maniobra, aunque señalamos que estos indicadores necesitan ser trabajados para volverlos operacionales. En segundo lugar, sostenemos que para desarrollar conocimientos sobre la intervención se necesita describir precisamente el proceso de intervención y tomar en cuenta el contexto en el que se desarrolla, por ello, la evaluación de sus efectos es insuficiente para desarrollar conocimientos sobre la intervención. Por último, la discusión señala que, desde nuestra perspectiva, la evaluación de las intervenciones constituye un proyecto en sí y que este contribuye directamente a la transformación del trabajo.

L'amélioration des pratiques d'intervention constitue aujourd'hui un enjeu certain dans le champ de la prévention des Troubles Musculo-Squelettiques (TMS) liés au travail. Le développement des connaissances concernant l'intervention en milieu de travail est donc un enjeu de recherche, qui convoque la thématique de l'évaluation des interventions. La recherche internationale dans le champ des TMS concentre largement ses travaux et recommandations sur l'évaluation des effets des interventions par l'évolution de l'état des symptômes des travailleurs concernés et/ou l'évolution des expositions. Dans ce texte, nous montrons tout d'abord que cette orientation suppose de réunir un certain nombre de conditions importantes, souvent difficiles à réunir et généralement sous-estimées. Nous proposons la prise en compte d'indicateurs relatifs à l'activité de travail et aux marges de manoeuvre des salariés, comme critères complémentaires de l'évaluation, tout en soulignant la nécessité d'un travail à réaliser pour les rendre opérationnels. Dans un deuxième temps, nous rappelons que l'évaluation des effets de 
l'intervention est insuffisante au développement de connaissances sur l'intervention : celui-ci suppose nécessairement la description précise du processus de l'intervention et la prise en compte du contexte de son déroulement. Enfin, la discussion souligne le fait que, dans notre perspective, l'évaluation des interventions constitue un projet en soi, qui contribue directement à la transformation du travail.

The improvement of intervention practices is today an undeniable issue in the field of workrelated musculoskeletal disorder (MSD) prevention. The development of knowledge about workplace interventions is therefore a research issue, which deals with the theme of intervention evaluation. International research in the MSD field focuses its work and recommendations mainly on the evaluation of the effects of interventions through the evolution in the state of the symptoms of the workers involved and/or the evolution in the exposures. In this article, we show first that this orientation assumes that a certain number of important conditions are associated, which are often difficult to associate and generally underestimated. We suggest that indicators relating to the work activity be considered, as well as employees' margins of maneuver, as additional criteria in the evaluation, while emphasizing the necessity that work be carried out to make them operational. Second, we reiterate that the evaluation of the intervention's effects is insufficient for developing knowledge about the intervention: this necessarily assumes the precise description of the intervention process and the consideration of its execution context. Finally, the discussion underlines the fact that, from our perspective, intervention evaluation is a project in itself, contributing directly to the transformation of the work.

\section{ÍNDICE}

Mots-clés: ergonomie, troubles musculo-squelettiques, formation, transmission, savoirs Keywords: work related musculoskeletal disorders, intervention assessment, intervention research criteria

Palavras-chave: problemas músculo-esqueléticos ligados ao trabalho, avaliação da intervenção, critérios de intervenção em investigação

Palabras claves: Lesiones músculo esqueléticas relacionadas con el trabajo, evaluación de la intervención, criterios de investigación sobre la intervención

\section{AUTORES}

\section{FABIEN COUTAREL}

Clermont Université, Université Blaise Pascal, 63000 Clermont-Ferrand, France

fabien.coutarel@univ-bpclermont.fr

\section{NICOLE VÉZINA}

CINBIOSE, Institut de santé et société, UQAM, Montréal, Canada

vezina.nicole@uqam.ca

\section{DIANE BERTHELETTE}

Département d'organisation et de ressources humaines, Institut de Santé et société, UQAM, Montréal, Canada

berthelette.diane@uqam.ca 


\section{AGNÈS AUBLET-CUVELIER}

Laboratoire d'Ergonomie et de biomécanique, INRS, Nancy, France agnes.aublet-cuvelier@inrs.fr

\section{ALEXIS DESCATHA}

INSERM, Villejuif, France

alexis.descatha@rpc.aphp.fr

\section{KARINE CHASSAING}

Département d'Ergonomie, IDC, Université Bordeaux2, 33076 Bordeaux, France

karine.chassaing@ergo.u-bordeaux2.fr

\section{YVES ROQUELAURE}

Laboratoire d'Ergonomie et d'Epidémiologie en Santé au Travail, CHU Angers, 49933 Angers,

France

yvroquelaure@chu-angers.fr

\section{CATHERINE HA}

Institut National de Veille Sanitaire, 94415 Saint-Maurice, France

c.ha@invs.sante.fr 